\title{
Articles
}

\author{
SONIA PÉREZ TOLEDO ${ }^{1}$ \\ VICENT SANZ ROZALÉN ${ }^{2}$
}

\section{Mujeres, trabajo, educación y control social en el siglo XXI}

\author{
Women, Work, Education and Social Control \\ in the 21st Century
}

Mujeres, trabajo o empleo, educación, control y subordinación, son temas centrales que se abordan en este dossier, el problema medular sin duda es la desigualdad de género en sus diversas expresiones. Como se señaló en un trabajo reciente, es un hecho que «a pesar del fuerte ingreso de las mujeres al espacio público y los logros obtenidos en términos de reformas legales, lo cierto es que, [...] el acceso a determinados derechos por parte de las mujeres, a garantías institucionales contra cualquier tipo de discriminación o violencia» (SMIRAGLIA, 2015) ha contribuido en los últimos tiempos a reducir en parte algunas de las forma en las que se expresan la subordinación y la desigualdad, pero ello no es suficiente pues estas resultan de procesos y fenómenos complejos de larga data.

Como lo señalan algunos de estudiosos de la historia de género, el terreno de las mentalidades y las prácticas sociales que forman parte y resultan de ellas, corresponden a la larga periodización y al ámbito de la cultura cuyas transformaciones son también lentas. Como lo muestran algunos de los trabajos aquí reunidos, las relaciones asimétricas, jerárquicas y de subordinación entre hombres y mujeres están entreveradas a un conjunto de elementos de entre los cuales el género y la sexualidad se articulan con factores tales como la raza o el origen, la posición social o de clase que, en su conjunto, contribuyen a limitar los alcances de las leyes y acciones positivas que buscan romper con la subordinación, la desigualdad y la violencia. Tal y como lo muestran los autores que colaboran en este número.

Desde esta perspectiva, no resulta sorprendente que se considere necesario estudiar problemas específicos que contribuyan a visibilizar la persistencia de las distintas formas y grados de inequidad de género, que en los últimos tiempos (incluidos los tiempos de pandemia) se ha expresado en algunos países un incremento de la violencia a pesar de la creación de instituciones y leyes dedicadas expresamente al combate a la discriminación y violencia de género. En el caso mexicano, solo como botón de muestra, tenemos los datos que resultan de un par de investigacio- 
nes recientes que indican un alto grado de desigualdad en los ámbitos del servicio público o el académico.

Al respecto, el estudio del Instituto Mexicano para la Competitividad A.C. publicó en el mes de mayo de este año los resultados de una amplia investigación indican que, si bien para alcanzar mayor igualdad de género en la vida pública en México se han establecido "cuotas de género» que han permitido, por ejemplo, el establecimiento de un Congreso paritario o bien el nombramiento de diecinueve mujeres al frente de Secretarías de Estado, este importante avance no tiene su correlato en un incremento en la participación económica de las mujeres, ya que «la presencia de las mujeres al interior de las secretarías de Estado disminuye conforme se eleva el puesto y nivel de ingresos», además de que «la brecha salarial dentro de la APF [Administración Pública Federal] es de 26\%» a pesar de que las mujeres tienen mayor nivel de escolaridad (TORRES, 2021).

Otra de las expresiones de la desigualdad de las mujeres se encuentra en el ámbito académico, en este caso se expresa entre otras formas en lo que la autora Fanny del Río denomina la «injusticia epistémica», concepto que le permite explicar la subrepresentación de las mujeres en el campo de la historia de la filosofía (DEL RÍO, 2020). En este aspecto, la autora demuestra como las filosofas mexicanas están desdibujadas en desarrollo de la historia de la filosofía, y lo documenta a partir del análisis de diecisiete obras sobre historia de la filosofía producidas en los últimos setenta años. Un análisis similar de la participación de las mujeres en los niveles más altos del Sistema Nacional de Investigadores de México o bien de las Academias Mexicanas también muestran una menor participación de las mujeres. Los casos referidos líneas arriba sin duda merecen mayores análisis, pero es evidente que comporten algunas de las preocupaciones formuladas por los autores de las investigaciones del Dossier.

Este número de ASPARKIA está integrado por ocho ensayos que se ocupan del estudio de mujeres en diferentes latitudes y realidades: América Latina, México, España, Ecuador y Colombia en el periodo reciente. Por su parte, en «Mujeres al mando: Cuotas de género y representación descriptiva de las mujeres en América Latina, Verónica Slaviero atiende el estudio de la participación política de las mujeres en varios países del continente americano. Se tarta de un análisis sobre el impacto de las cuotas de género como acción afirmativa para incrementar la representación femenina durante el periodo transcurrido en las tres últimas décadas en Argentina, Bolivia, Brasil, Chile, Colombia, Costa Rica, Ecuador, El Salvador, Honduras, México, Nicaragua, Perú y Uruguay a partir del estudio de las leyes electorales de cada uno de los países, así como de la estructura de la las papeletas electorales a partir de un análisis estadístico que le permite llegar a conclusiones relativas al incremento de la participación de las mujeres en aquellos países que han establecido una política de acciones afirmativas, sin perder de vista las posturas discriminatorias y evasivas a lo largo del periodo estudiado.

En el tercer trabajo denominado «México: el reto de ser mujer dentro de una estructura patriarcal» de Fermina Martínez Rivera y Emmanuel Díaz del Ángel, los autores abordan el acoso sexual callejero y para ello parten de la caracterización 
del acoso y su normalización. Los autores presentan una apretada síntesis sobre temas muy amplios y complejos que han dado pie a debates importantes. Así, género, feminismo tanto como los distintos tipos de acoso se constituyen en el marco para aportar datos que permiten observar la problemática del acoso a través de la presentación de algunas cifras indicativas de la violencia y acoso sexual en mujeres y niñas en México. Para concluir con una revisión de la producción o estado del arte relativo al tema del acoso callejero y en particular sobre la vulnerabilidad que enfrentan las mujeres en el espacio público a pesar de los discursos y las leyes que pretenden eliminar estas conductas.

Ahora bien, Lorena Acosta Reveles introduce un «Científicas a la sombra, también en el espacio virtual», la problemática a la que nos hemos referido con al inicio, la prevalencia de la desigualdad en liderazgo y salarial que muestra las estructuras patriarcales en el trabajo científico en universidades mexicanas. En este ensayo la autora desarrolla su argumentación a partir de la evidencia empírica que arroja el estudio de repositorios de acervos académicos en internet, lo cual le permite concluir que «la presencia y la posición jerárquica de las investigadoras, son aún más opacas en internet que en el espacio físico-institucional.» Toma en consideración el planteamiento de una menor participación de las mujeres en el Sistema Nacional de Investigadores como lo indicamos antes, pues sólo reporta una proporción femenina integrada por 37 puntos respecto del total de miembros del sistema, así como adscripción a los niveles más bajos que indican, de acuerdo con la autora del artículo, un problema de segregación con cifras contundentes a la luz de la información correspondiente a 2019. En ese sentido, no resulta sorprendente que desde las primeras páginas se reconozcan los avances y la implementación de políticas particulares que buscan impulsar la participación femenina en la ciencia desde el Consejo Nacional de Ciencia y Tecnología mexicano, pero sin dejar de señalar que «el camino hacia la inclusión y la equidad de las mujeres en ciencias y en el reconocimiento de sus méritos se avizora todavía largo y tortuoso.»

El ensayo relativo al estudio del «Género, formación profesional e inserción laboral» que aparece en quinto lugar, la autora se ocupa de la España actual, busca explicar la brecha entre la formación profesional y mercado laboral. En él Ana María Moreno Márquez considera que «En el Plan Estratégico de Formación Profesional se indica que «en pleno siglo XXI no hay ninguna razón para que determinadas profesiones estén ocupadas mayoritariamente por mujeres y otras por hombres», por ello, es importante analizar por qué se produce esta situación.». Es un ensayo que se ocupa de los tiempos recientes con una perspectiva más amplia que incluye, aunque no lo indique el título, una reflexión breve sobre el papel de educación y la familia en la reproducción de los «roles de género tradicionales» que inciden en la segregación horizontal y vertical que se pueden observar tanto en la formación profesional (lo que da como resultado la feminización de un tipo particular de carreras de formación profesional frente a otras prácticamente masculinizadas), así como en las posibilidades de acceso al mercado de trabajo. Un mercado de trabajo que sigue siendo más restringido para las mujeres y cuyos salarios siguen siendo menor respecto de los hombres, asunto al que nos referimos en las primeras páginas 
de esta breve presentación. En este sentido, a partir del estudio de la legislación en contraste con la información empírica disponible se muestra la distancia entre las intenciones del legislador en materia de igualdad y equidad género respecto de las prácticas.

Rosa María García introduce un elemento fundamental el estudio de la migración, tema central en nuestras sociedades tanto en el pasado como en presente, para abordar el explicar la doble segregación que enfrentan las mujeres migrantes en España, en particular en Murcia, y para las cuales «el techo de cristal» es aún más evidente al igual que la feminización de las ocupaciones, pero no sólo a partir de esta diferencia, sino de la que introduce el lugar de origen o procedencia de las migrantes. En otras palabras, el estudio de Rosa María García muestra que el tipo de actividades a las que se vincula la población femenina migrante está relacionado con el servicio doméstico, el trabajo de cuidados, así como el trabajo sexual. Asimismo, en el ensayo su autora llama la atención acerca de una mayor vulnerabilidad de este grupo de mujeres en tanto migrantes, condición que se agrega a la situación de desigualdad propia del tipo de trabajo al que pueden aspirar y, por supuesto, a la diferenciación salarial que, como vimos, no es privativa del trabajo poco calificado como el servicio doméstico sino también está presente en otros ámbitos laborales al que nos hemos referido antes, como el de las mujeres que laboran en la Administración Pública Federal mexicana o de las científicas que participan en el Sistema Nacional de Investigadores en los niveles inferiores de mando o de categoría como investigador, pues en ambos casos la brecha salarial entre hombres y mujeres es evidente. En todos casos la desigualdad es imperante, pero que duda cabe que la condición de migrante la exacerba y abre la posibilidad a un mayor control y subordinación, así como a diversos tipos de violencia.

Los artículos con los que se cierra este dossier estudian a mujeres de condiciones particulares de violencia y sujeción. El de «La violencia sexual contra la mujer en zonas de conflicto armado en Colombia: un enfoque a partir de un modelo logit» de la autoría de Katerina Tinjaca Uriza y María Lourdes Santos Pérez, por un lado; y, por el otro, el de Virginia Ballester Martínez que realiza un acercamiento cualitativo con métodos etnográficos a reclusas de la cárcel de Santa Martha Acatitla ubicada en el oriente de la Ciudad de México. En relación con el primero de los textos, las autoras analizan la violencia sexual contra las mujeres en las regiones colombianas afectadas por la guerra. A partir del análisis estadístico de la encuesta ENVISE se proponen identificar los factores asociados a la probabilidad de ser víctima de violencia sexual, teniendo en cuenta los factores específicos mostrados por la propia fuente. Por su parte, en «Mujeres en resistencia: estrategias subversivas en la cárcel de Santa Martha Acatitla, Ciudad de México» Virginia Ballester parte del estudio de la información que le aporta las entrevistas realizadas a trece reclusas con la finalidad de estudiar, como lo indica el título del artículo, las estrategias subversivas a las que recurren las mujeres privadas de su libertad. Como punto de partida la autora ofrece una caracterización del sistema carcelario introduciendo al análisis conceptos como el de «infra política» o de «discursos ocultos» formulados por J, Scott, así como los mecanismos y prácticas de control corporal y sujeción 
propios de los sistemas carcelarios, a partir de lo cual se propuso el estudio de trece mujeres que participaron de forma voluntaria en el denominado por la autora «Taller de Género y Resistencias». Mediante la aplicación de métodos cualitativos, como la observación participante y las entrevistas programadas, Ballester informa acerca de las dificultades que que enfrentan estas mujeres para resistir al control, la subordinación y el castigo. Asimismo, señala que las diferencias que se establecen entre las mismas reclusas respecto de, por ejemplo, la maternidad o bien de los roles tradicionales relativos al «buen» comportamiento femenino o el reducido margen de acción para los comportamientos contestatarios.

En definitiva, un conjunto de trabajos que, desde diferentes perspectivas, con diferentes metodologías y desde distintas disciplinas, ofrece una panorámica de amplio espectro sobre la realidad de las mujeres en América Latina y España en estos inicios del siglo xxI.

\section{Bibliografía}

DEL RÍO, F., Hacia una crítica ética de la historia de la Filosofía en México desde una perspectiva de género, México, UNAM, 2020.

SMIRAGLIA. Romina, «Feminismo y Liberalismo: una revisión de El Contrato Sexual de Carole Pateman», Leviathan. Cuadernos de Investigación Política, núm. 11, 2015.

TORRES, M. (coord.), Mujeres en la Administración Pública Federal: más allá de la foto. México, Instituto Mexicano para la Competitividad A.C., 2021. 